\title{
Correspondence
}

\section{New Design and Realization Techniques for a Class of Perfect Reconstruction Two-Channel FIR Filterbanks and Wavelets Bases}

\author{
S. C. Chan, Carson K. S. Pun, and K. L. Ho
}

\begin{abstract}
This paper proposes two new methods for designing a class of two-channel perfect reconstruction (PR) finite impulse response (FIR) filterbanks (FBs) and wavelets with $K$-regularity of high order and studies its multiplier-less implementation. It is based on the two-channel structural PR FB proposed by Phoong et al. The basic principle is to represent the $K$-regularity condition as a set of linear equality constraints in the design variables so that the least square and minimax design problems can be solved, respectively, as a quadratic programming problem with linear equality constraints (QPLC) and a semidefinite programming (SDP) problem. We also demonstrate that it is always possible to realize such FBs with sum-of-powers-of-two (SOPOT) coefficients while preserving the regularity constraints using Bernstein polynomials. However, this implementation usually requires long coefficient wordlength and another direct-form implementation, which can realize multiplier-less wavelets with $K$-regularity condition up to fifth order, is proposed. Several design examples are given to demonstrate the effectiveness of the proposed methods.
\end{abstract}

Index Terms-Dyadic wavelets, multiplier-less realization, perfect reconstruction filterbanks, regularity, semidefinite programming.

\section{INTRODUCTION}

Perfect reconstruction (PR) multirate filterbanks (FBs) have important applications in signal analysis, signal coding, and the design of wavelet bases. Similar to the lifting scheme [10], the structural PR two-channel finite impulse response (FIR) FBs proposed in [1] are particularly attractive because the PR property is structurally imposed, and their design and implementation complexities are very low. The FBs are parameterized by two functions $\beta(z)$ and $\alpha(z)$ and some delay parameters. These two functions can be chosen as linear-phase FIR or allpass functions to realize FIR and IIR FBs with very good frequency characteristics. In [2], the design of these structural PR two-channel FBs was formulated as a complex Chebyshev approximation problem, which was solved using the REMEZ exchange algorithm to obtain linear-phase as well as low-delay FBs. The construction of wavelet bases from these structural PR FBs having the maximally possible $K$-regularity condition was first considered in [1] with identical functions $\beta(z)$ and $\alpha(z)$. Later, in [2], the construction of low-delay wavelet bases satisfying the $K$-regularity condition up to one zero-order moment was also studied. Due to the difficulty in solving the constrained optimization problem, the incorporation of $K$-regularity with higher order, however, was not addressed. The design of paraunitary twochannel FIR FBs [the conjugate quadrature filters (CQF) [8]] with a prescribed number of $K$-regularity was previously considered in [9] The wavelet FBs so obtained are in general not linear phase. Recently,

Manuscript received April 11, 2003; revised August 26, 2003. Part of this work was presented at the 14th International Conference on Digital Signal Processing, Santorini, Greece, July, 2002 [5]. The associate editor coordinating the review of this manuscript and approving it for publication was Dr. Anamitra Makur.

The authors are with the Department of Electrical and Electronic Engineering, The University of Hong Kong, Hong Kong (e-mail: scchan@eee. hku.hk; kspun@eee.hku.hk; klho@eee.hku.hk).

Digital Object Identifier 10.1109/TSP.2004.828918
Tay [11] showed that it is possible to incorporate the $K$-regularity condition for the linear-phase FBs in [1] using the Bernstein polynomial. Since the error function is a linear function of the coefficients in the Bernstein polynomial, it can be solved as a simple quadratic programming problem for the least squares error criterion. The genetic algorithm (GA) was also proposed for designing such FBs and wavelets with higher order moments and sum-of-powers-of-two (SOPOT) coefficients [3]. In this paper, we extend the work in [2] to the case of higher order $K$-regularities [5]. This will enable a larger class of wavelet FBs with different smoothness and delay to be constructed. More precisely, we show that it is possible to formulate the least square and minimax design problems, respectively, as a quadratic programming problem with linear equality constraints (QPLC) and an semidefinite programming (SDP) problem [15]. The approach is rather general, and it applies to the linear-phase as well as the low-delay cases without making use of the Bernstein polynomial expansion. The design of linear-phase structural PR two-channel FBs and wavelets was recently reported in [24], and the present work will be focused on the low-delay situation. In addition, we will show that by employing the Bernstein polynomial form of digital filters, it is always possible to construct wavelet bases with SOPOT coefficients. Our Bernstein polynomial representation for halfband filters can be viewed as a generalization (prescribed flatness instead of maximally flat) of the low-delay maximally flat halfband filter that was recently proposed in [19]. Unfortunately, the complexity of this direct-form implementation is usually very high due to the long wordlength requirement of the expanded coefficients. We therefore propose another simpler factorization, which is capable of realizing SOPOT wavelet bases up to fifth order of $K$-regularity at a much lower wordlength. The multiplier-less realization of the resulting FB and wavelets using SOPOT coefficients and a multiplier block (MB) [13] are also described. The paper is organized as follows: Section II is devoted to the proposed QPLC and SDP design methods. The multiplier-less realization of the wavelets FB is given in Section III. Conclusions are drawn in Section IV.

\section{Design of the Two-Channel Structural PR FBs}

The structural PR FBs [1], [2], as shown in Fig. 1, are parameterized by subfilter pairs $\beta(z)$ and $\alpha(z)$ and two delay parameters $N$ and $M$. The FB is PR for an arbitrary choice of filter pairs $\beta(z)$ and $\alpha(z)$, and the frequency responses of the analysis filters are given by

$$
\begin{aligned}
& H_{0}\left(e^{j \omega}\right)=\frac{1}{2}\left(e^{-j 2 N \omega}+e^{-j \omega} \beta\left(e^{j 2 \omega}\right)\right) \\
& H_{1}\left(e^{j \omega}\right)=-\alpha\left(e^{j 2 \omega}\right) H_{0}\left(e^{j \omega}\right)+e^{j(-2 M-1) \omega} .
\end{aligned}
$$

Let $\beta\left(e^{j \omega}\right)$ and $\alpha\left(e^{j \omega}\right)$ be FIR filter with lengths $L_{\beta}$ and $L_{\alpha}$, respectively. In [2], the desired responses of $\beta\left(e^{j \omega}\right)$ and $\alpha\left(e^{j \omega}\right)$ are studied. It can be shown that the ideal responses of $\beta\left(e^{j \omega}\right)$ and $\alpha\left(e^{j \omega}\right)$ are given, respectively, by

$$
\begin{aligned}
\beta_{d}\left(e^{j \omega}\right) & =e^{j(-N+1 / 2) \omega} \\
\alpha_{d}\left(e^{j \omega}\right) & =\frac{e^{j(-M-1 / 2) \omega}}{H_{0}\left(e^{j \omega / 2}\right)}, \quad \omega \in[-\pi, \pi] .
\end{aligned}
$$

It can be seen that $\beta\left(e^{j 2 \omega}\right)$ approximates $e^{-j(2 N-1) \omega}$ and $-e^{-j(2 N-1) \omega}$ for $0<\omega<\pi / 2$ and $\pi / 2<\omega<\pi$, respectively. Hence, $H_{0}\left(e^{j \omega}\right)$ approximates $e^{-j(2 N-1) \omega}$ in the passband and becomes zero for $\pi / 2<\omega<\pi$. As for $\alpha\left(e^{j 2 \omega}\right)$, the product 

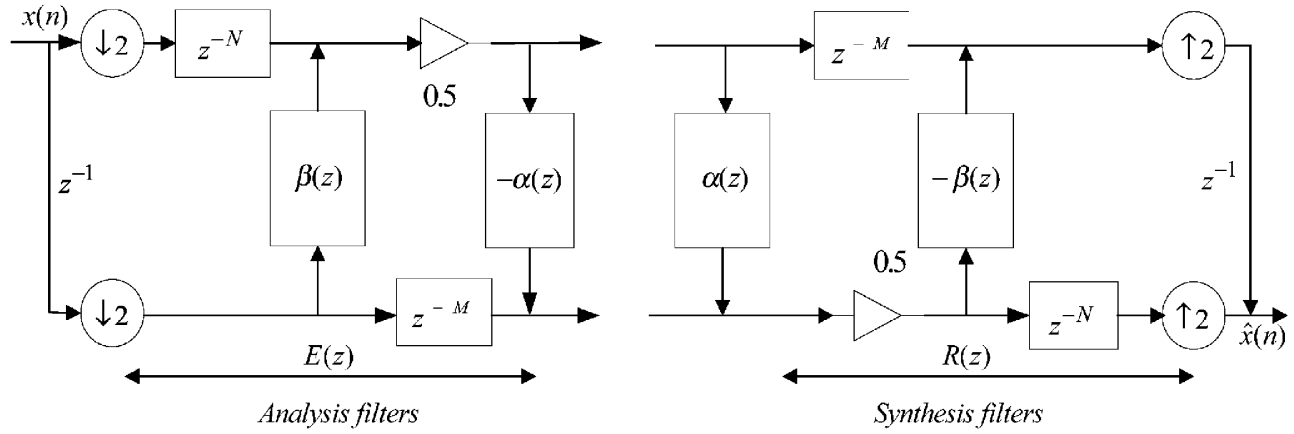

Fig. 1. Two-channel structural PR FB in [1].

$H_{0}\left(e^{j \omega}\right) \alpha\left(e^{j 2 \omega}\right)$ will approximate $e^{j(-2 M-1) \omega}$ in $0<\omega<\pi / 2$ and is nearly zero in the stopband because of $H_{0}\left(e^{j \omega}\right)$. Thus, $H_{1}\left(e^{j \omega}\right)$ will be close to $e^{j(-2 M-1) \omega}$ in the passband and zero in the stopband. To construct a wavelet FB, the analysis filters $H_{0}\left(e^{j \omega}\right)$ and $H_{1}\left(e^{j \omega}\right)$ should satisfy a certain $K$-regularity condition. More precisely, $H_{0}\left(e^{j \omega}\right)$ and $H_{1}\left(e^{j \omega}\right)$ should possess at least one zero at $\omega=\pi$ and $\omega=0$, respectively. Next, we will express this $K$-regularity condition as a set of linear equality constraints so that the design problem can be solved as a QPLC or an SDP problem. If the least squares (LS) criterion is used to approximate the desired responses in (2), the objective functions for $\beta\left(e^{j \omega}\right)$ and $\alpha\left(e^{j \omega}\right)$ are

$$
\begin{aligned}
& L_{2}(\boldsymbol{\beta})=\int_{-2 \omega_{\beta p}}^{2 \omega_{\beta p}} \boldsymbol{W}(\omega)\left|\beta^{T} \cdot e_{L_{\beta}}-\beta_{d}\left(e^{j \omega}\right)\right|^{2} d \omega \\
& L_{2}(\boldsymbol{\alpha})=\int_{-2 \omega_{\alpha p}}^{2 \omega_{\alpha p}} \boldsymbol{W}(\omega)\left|\boldsymbol{\alpha}^{T} \cdot e_{L_{\alpha}}-\alpha_{d}\left(e^{j \omega}\right)\right|^{2} d \omega
\end{aligned}
$$

where $\boldsymbol{e}_{q}=\left[\begin{array}{llll}1 & e^{-j \omega} & \cdots & e^{-j(q-1) \omega}\end{array}\right]^{T} \cdot \omega_{\beta p}$ and $\omega_{\alpha p}$ are the passband and stopband cut-off frequencies of $H_{0}\left(e^{j \omega}\right)$ and $H_{1}\left(e^{j \omega}\right)$, respectively. $\beta=\left[\begin{array}{llll}\beta_{0} & \beta_{1} & \ldots & \beta_{L_{\beta}-1}\end{array}\right]^{T}$ and $\boldsymbol{\alpha}=\left[\begin{array}{llll}\alpha_{0} & \alpha_{1} & \ldots & \alpha_{L_{\alpha}-1}\end{array}\right]^{T}$ are, respectively, the impulse responses of $\beta(z)$ and $\alpha(z)$, and $W(\omega)$ is a positive weighting function. Expanding (3), we have

$$
\begin{aligned}
& L_{2}(\boldsymbol{\beta})=\boldsymbol{\beta}^{T} \cdot \boldsymbol{W}_{\beta} \cdot \boldsymbol{\beta}-2 \boldsymbol{\beta}^{T} \cdot \boldsymbol{g}_{\beta}+\boldsymbol{p}_{\beta} \\
& L_{2}(\boldsymbol{\alpha})=\boldsymbol{\alpha}^{T} \cdot \boldsymbol{W}_{\alpha} \cdot \alpha-2 \boldsymbol{\alpha}^{T} \cdot \boldsymbol{g}_{\alpha}+\boldsymbol{p}_{\alpha}
\end{aligned}
$$

where

$$
\begin{aligned}
\boldsymbol{W}_{i} & =\int_{-2 \omega_{i p}}^{2 \omega_{i p}} W(\omega) \boldsymbol{e}_{L_{i}} \cdot \boldsymbol{e}_{L_{i}}^{H} d \omega \\
\boldsymbol{g}_{i} & =\int_{-2 \omega_{i p}}^{2 \omega_{i p}} \boldsymbol{R} \boldsymbol{e}\left(W(\omega) \boldsymbol{e}_{L_{i}} \cdot i_{d}^{*}\left(e^{j \omega}\right)\right) d \omega
\end{aligned}
$$

and $\boldsymbol{p}_{i}$ are some constants with $i=\alpha, \beta$. The subscript $\boldsymbol{H}$ denotes Hermitian transpose. The optimal least squares solution is obtained by differentiating (4) with respect to the optimization vector variables and setting the resulting derivatives to zero. It gives

$$
\beta_{o p t}=\boldsymbol{W}_{\beta}^{-1} \cdot \boldsymbol{g}_{\beta} \text { and } \boldsymbol{\alpha}_{o p t}=\boldsymbol{W}_{\alpha}^{-1} \cdot \boldsymbol{g}_{\alpha} .
$$

As mentioned previously, a wavelet FB can be constructed from the analysis filters if $H_{0}\left(e^{j \omega}\right)$ and $H_{1}\left(e^{j \omega}\right)$ possess at least one zero at $\omega=\pi$ and $\omega=0$, respectively. A higher order of $K$-regularity is obtained if $K_{0}$ and $K_{1}(>1)$ zeros are imposed at $\omega=\pi$ and $\omega=0$ for $H_{0}\left(e^{j \omega}\right)$ and $H_{1}\left(e^{j \omega}\right)$, respectively. Note that $K_{0}$ should be greater or equal to $K_{1}>1$. This is equivalent to $\left[\left(d^{k_{0}} / d \omega^{k_{0}}\right) H_{0}\left(e^{j \omega}\right)\right]_{\omega=\pi}=\left[\left(d^{k_{1}} / d \omega^{k_{1}}\right) H_{1}\left(e^{j \omega}\right)\right]_{\omega=0}=0$, for $k_{0}=0, \ldots, K_{0}-1$ and $k_{1}=0, \ldots, K_{1}-1$. When incorporating the regularity constraints for $H_{1}\left(e^{j \omega}\right)$, it is assumed that a regularity of order $K_{0}$ has already been incorporated in $H_{0}\left(e^{j \omega}\right)$. After performing the differentiation, it can be shown that the regularity constraints [25] can be expressed in terms of the coefficients of $\beta(z)$ and $\alpha(z)\left(\beta_{m}\right.$ and $\left.\alpha_{m}\right)$ as in (6), shown at the bottom of the page, for $k_{0}=0, \ldots, K_{0}-1$ and $k_{1}=0, \ldots, K_{1}-1$. $\lceil x\rceil$ denotes the integer that is just greater than or equal to $x$. Note that (6) is a set of linear equality constraints in $\beta$ and $\boldsymbol{\alpha}$, and it can also be written as $\boldsymbol{B} \cdot \boldsymbol{\beta}=\boldsymbol{c}_{\beta, K_{0}}$ and $\boldsymbol{A} \cdot \boldsymbol{\alpha}=\boldsymbol{c}_{\alpha, K_{1}}$, for some matrices $\boldsymbol{B}$ and $\boldsymbol{A}$, and vectors $\boldsymbol{c}_{\beta, K_{0}}$ and $\boldsymbol{c}_{\alpha, K_{1}}$, whose entries are defined in (6). Equations (5) and (6) together form a quadratic programming problem subject to a set of linear equality constraints, which can be solved analytically using the method of Lagrange multiplier [5], [16]. The solution is

$$
\begin{aligned}
\boldsymbol{\beta}_{o p t}= & \boldsymbol{W}_{\beta}^{-1} \cdot \boldsymbol{g}_{\beta}-\frac{1}{2} \boldsymbol{W}_{\beta}^{-1} \cdot \boldsymbol{\lambda}_{\beta}^{T} \cdot \boldsymbol{B} \\
\boldsymbol{\alpha}_{o p t}= & \boldsymbol{W}_{\alpha}^{-1} \cdot \boldsymbol{g}_{\alpha}-\frac{1}{2} \boldsymbol{W}_{\alpha}^{-1} \cdot \boldsymbol{\lambda}_{\alpha}^{T} \cdot \boldsymbol{A} \\
\text { where } \boldsymbol{\lambda}_{\beta}= & 2\left(\boldsymbol{B} \boldsymbol{B}^{T}\right)^{-1}\left(\boldsymbol{g}_{\beta}-\boldsymbol{W}_{\beta}\left(\boldsymbol{B}^{T} \boldsymbol{B}\right)^{-1} \boldsymbol{B}^{T} \boldsymbol{c}_{\beta, k_{0}}\right)^{T} \\
\boldsymbol{\lambda}_{\alpha}= & 2\left(\boldsymbol{A} \boldsymbol{A}^{T}\right)^{-1} \\
& \times\left(\boldsymbol{g}_{\alpha}-\boldsymbol{W}_{\alpha}\left(\boldsymbol{A}^{T} \boldsymbol{A}\right)^{-1} \boldsymbol{A}^{T} \boldsymbol{c}_{\alpha, k_{1}}\right)^{T} .
\end{aligned}
$$

$$
\begin{aligned}
\sum_{m=0}^{L_{\beta}-1-\left\lceil k_{0} / 2\right\rceil} \beta_{m} \frac{\left(2\left(L_{\beta}-m\right)-2\right) !}{\left(2\left(L_{\beta}-m\right)-2-k_{0}\right) !} & =\frac{\left(2\left(L_{\beta}-N\right)-1\right) !}{\left(2\left(L_{\beta}-N\right)-1-k_{0}\right) !} \\
& \sum_{r=0}^{k_{1}}\left(\sum_{m=0}^{L_{\alpha}-1-\left\lceil\left(k_{1}-r\right) / 2\right\rceil} \alpha_{m} \frac{C_{r}^{k_{1}}\left(2\left(L_{\beta}-N\right)-1\right) !\left(-2 m+2 L_{\alpha}-2\right) !}{\left(2\left(L_{\beta}-N\right)-1-r\right) !\left(-2 m+2 L_{\alpha}-2-k_{1}+r\right) !}\right) \\
& =\frac{\left(2\left(L_{\alpha}+L_{\beta}-M\right)-4\right) !}{\left(2\left(L_{\alpha}+L_{\beta}-M\right)-4-k_{1}\right) !}
\end{aligned}
$$


$\beta(z)$ and $\alpha(z)$ can also be designed with a minimax design criterion and a prescribed $K$-regularity using SDP. First of all, let us consider the design of $\beta(z)$. To minimize the maximum ripple of the approximation error is equivalent to the following:

$$
\min _{\beta} \max \left|\beta^{T} \cdot e-\beta_{d}\left(e^{j \omega}\right)\right|^{2}, \text { for } \omega \in\left[0,2 \omega_{\beta p}\right]
$$

To solve (8) using the SDP, we densely discretize $\omega$ over the band of interest $\omega \in\left[0,2 \omega_{\beta p}\right]$ into a set of frequencies points $\omega_{k}$ 's, $k=1, \ldots K$. The optimization problem (9) is then approximated as

$$
\begin{aligned}
& \min \delta \\
& \eta_{R, \beta}^{2}\left(\omega_{k}\right)+\eta_{I, \beta}^{2}\left(\omega_{k}\right) \leq \delta, \quad k=1, \ldots, K \\
& \eta_{I, \beta}(\omega)=\boldsymbol{\beta}^{T} \cdot \boldsymbol{s}+\boldsymbol{I m}\left\{\beta_{d}\left(e^{j \omega}\right)\right\} \\
& \eta_{R, \beta}(\omega)=\boldsymbol{\beta}^{T} \cdot \boldsymbol{c}-\boldsymbol{R} \boldsymbol{R}\left\{\beta_{d}\left(e^{j \omega}\right)\right\} \\
& \boldsymbol{c}=\left[\begin{array}{llll}
1 & \cos (1 \cdot \omega) & \cdots & \cos \left(\left(L_{\beta}-1\right) \cdot \omega\right)
\end{array}\right]^{T} \\
& \boldsymbol{s}=\left[\begin{array}{llll}
0 & \sin (1 \cdot \omega) & \cdots & \sin \left(\left(L_{\beta}-1\right) \cdot \omega\right)
\end{array}\right]^{T} .
\end{aligned}
$$

$\boldsymbol{R} \boldsymbol{e}\{x\}$ and $\boldsymbol{I m}\{x\}$ denote the real and imaginary parts of $x$, respectively. Using the Schur complement [15], it can be shown that (9) is equivalent to [14]

$$
\begin{aligned}
& \min \delta \\
& F_{k}(\beta) \geq 0, \quad k=1, \ldots, K \\
& F_{k}(\beta)=\left(\begin{array}{ccc}
\delta & \eta_{R, \beta}\left(\omega_{k}\right) & \eta_{I, \beta}\left(\omega_{k}\right) \\
\eta_{R, \beta}\left(\omega_{k}\right) & 1 & 0 \\
\eta_{I, \beta}\left(\omega_{k}\right) & 0 & 1
\end{array}\right)
\end{aligned}
$$

which is a set of linear matrix inequalities (LMIs) [15] and can be solved efficiently using SDP. The advantages of SDP are i) it is possible to determine whether a feasible solution exists, and if so, it is possible to determine the global optimal solution, since the problem is convex, and ii) linear (and certain type of nonlinear constraints) can be imposed. In order to simultaneously solve the SDP problem (10) and satisfy the regularity constraints in (6), the dependent variables defined by (6) are expressed as a linear combination of the independent variables and are subsequently eliminated. An alternative approach is to stack the constraints in (6) with the constraints in (10). See [5] and [25] for more details. The design of $\alpha(z)$ can be formulated similarly. Thanks to the use of SDP, the linearly constrained complex Chebyshev problem can be optimally solved. Traditionally, the optimal minimax design of linearly constrained linear-phase FIR filters is solved using linear programming [7], whereas the nonlinear-phase FIR case is less studied. The above problem can also be cast into a special case of SDP called the second-order cone programming problem (SOCP) for better computational efficiency. Due to space limitations, the details are omitted here.

Example 1: For comparison purposes, two-channel PR FBs having the same specification as in [2, Ex. 4.1] were designed. However, the number of zeros to be imposed for $H_{0}\left(e^{j \omega}\right)$ and $H_{1}\left(e^{j \omega}\right)$ are $K_{0}=2$ and $K_{1}=1$, respectively. The lengths of $\beta(z)$ and $\alpha(z)$ are, respectively, $L_{\beta}=8$ and $L_{\alpha}=10$, and the delay parameters are $N=2$ and $M=5$. The system delay is 15 samples. The passband cut-off frequencies of $H_{0}\left(e^{j \omega}\right)$ and $H_{1}\left(e^{j \omega}\right)$ are $0.34 \pi$ and $0.66 \pi$, respectively. The results for the QPLC method are shown in Fig. 2(a). For the SDP design method, $\omega$ in the passband is uniformly discretized using 500 samples. The optimization is carried out using the MATLAB LMI Toolbox, and it takes fewer than 60 iterations to obtain the solution. The design results are shown in Fig. 2(b) and (c) (the phase response of the QPLC method is similar and is omitted). The stopband attenuation of $H_{0}(z)$ is $41.5 \mathrm{~dB}$, whereas that of [2] is a bit less than $40 \mathrm{~dB}$. For $H_{1}(z)$, our design gives a stopband attenuation of $46.3 \mathrm{~dB}$, which is better than that in [2]. This 6-dB stopband attenuation gain is possibly due to the splitting of the Chebyshev approximation problem into two independent problems in [2], whereas the proposed SDP formulation minimizes the complex approximation error in the Chebyshev sense. The scaling and wavelets functions of the SDP method are shown in Fig. 2(d) and (e). Those for the QPLC method are very similar visually.

Example 2: In this example, a two-channel PR FB with $K_{0}=3$ and $K_{1}=3$ is designed. The lengths of $\beta(z)$ and $\alpha(z)$ are $L_{\beta}=14$ and $L_{\alpha}=12$, respectively, and the delay parameters are $N=4$ and $M=8$ (system delay $=25$ samples). The passband cut-off frequencies of $H_{0}\left(e^{j \omega}\right)$ and $H_{1}\left(e^{j \omega}\right)$ are 0.415 and $0.625 \pi$, respectively. Due to page limitations, only the design results for the SDP method are given. $\omega$ in the passband is uniformly discretized using 500 samples, and it takes fewer than 100 iterations to obtain the solution. The design result is shown in Fig. 3, and the stopband attenuations of $H_{0}(z)$ and $H_{1}(z)$ are 40.275 and $41 \mathrm{~dB}$, respectively. Note the small bump $(3 \mathrm{~dB})$ in the transition band, which is a typical phenomenon in low-delay FIR FBs. It is possible to suppress this bump by imposing additional linear constraints in the SDP formulation at the expense of lower stopband attenuation.

\section{Design of MultiPlier-Less Two-Channel WaVELETS FBS}

In Section II, we have illustrated how the $K$-regularity conditions can be incorporated numerically by the QPLC and SDP methods. In practical implementation of these wavelet FBs, the filter coefficients of $\beta(z)$ and $\alpha(z)$ have to be quantized, and the $K$-regularity condition might no longer hold. Here, we propose new realization techniques that preserve the $K$-regularity condition even when the filter coefficients are expressed as SOPOT coefficients of the form $b=\sum_{k=0}^{L-1} a_{k} \cdot 2^{-b_{k}}$, where $a_{k} \in\{-1,0,1\}$, and $b_{k} \in\left\{-l_{L}, \ldots 1,0, \ldots l_{U}\right\}$. The larger the numbers $l_{L}, l_{U}$, and $L$, the closer the SOPOT approximation will be to the original real number. In practice, the number of nonzero terms is usually kept to a small number, while satisfying the given specification, so that the multiplication can be implemented as a limited number of shift and add (subtract) operations, giving rise to multiplier-less realization. We first generalize an important result developed in [1] regarding the zeros of $H_{0}(z)$ and $F_{0}(z)$ when $\beta(z)$ is equal to $\alpha(z)$. More precisely, it is shown in the Appendix that the synthesis filter $F_{0}(z)$ is related to $H_{0}(z)$ as $F_{0}(z)=-\alpha\left(z^{2}\right) \cdot H_{0}(z)+2 H_{\alpha}(z) \cdot z^{-2 N+1}$, where $H_{\alpha}(z)=\left[z^{-1} \alpha\left(z^{2}\right)+z^{-2 N_{\Delta}}\right] / 2$, and $N_{\Delta}=(M-N)+1$. Since the common zeros of $H_{0}(z)$ and $H_{\alpha}(z)$ are also zeros of $F_{0}(z)$, if $H_{0}(z)$ and $H_{\alpha}(z)$ have $K_{1}$ common zeros at $z=-1$, then $F_{0}(z)$ has a $K$-regularity of order equal to or greater than $K_{1}$. To structurally impose these zeros at $z=-1$, one may express the halfband filters $H_{0}(z)=H_{\beta}(z)$ and $H_{\alpha}(z)$ using the Bernstein polynomial expansion as follows:

$$
\begin{aligned}
H_{0}(z) & =z^{-1} H(z) \\
& =z^{-1}\left(\sum_{i=0}^{L} b_{i}\left(\frac{1+z^{-1}}{2}\right)^{i}\left(\frac{1-z^{-1}}{2}\right)^{L-i}\right) \\
& =\frac{z^{-1}}{2^{L}}\left(\sum_{i=0}^{L} b_{i}\left(1+z^{-1}\right)^{i}\left(1-z^{-1}\right)^{L-i}\right)
\end{aligned}
$$

where $L$ is the order of $H(z)$, and $b_{i}$ s are the coefficients of the Bernstein polynomial expansion. It can be seen that if $H_{0}(z)$ has $K_{0}$ zeros at $z=-1$, then the coefficients $b_{i}$ for $i=0, \ldots, K_{0}-1$ will be zero. 


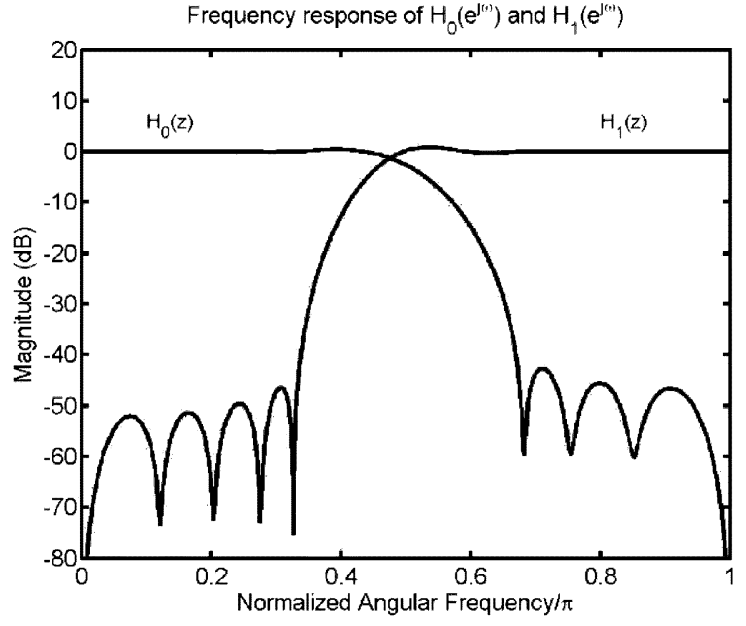

(a)

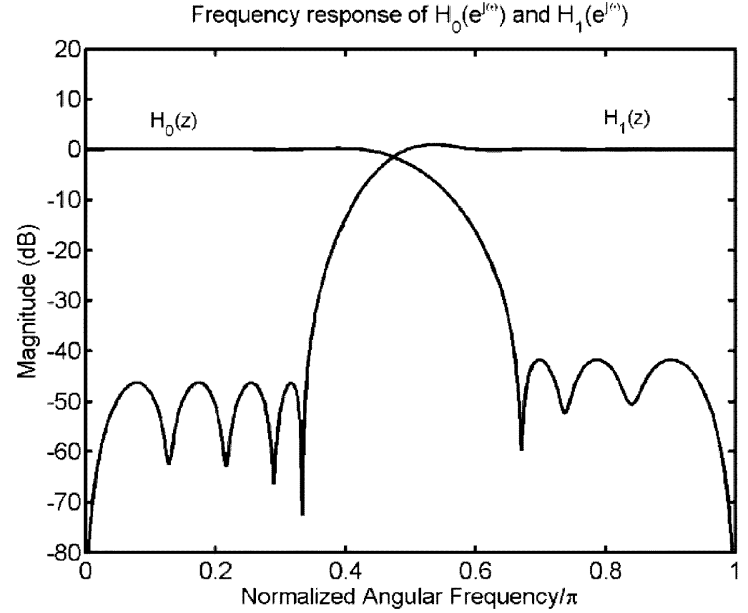

(b)

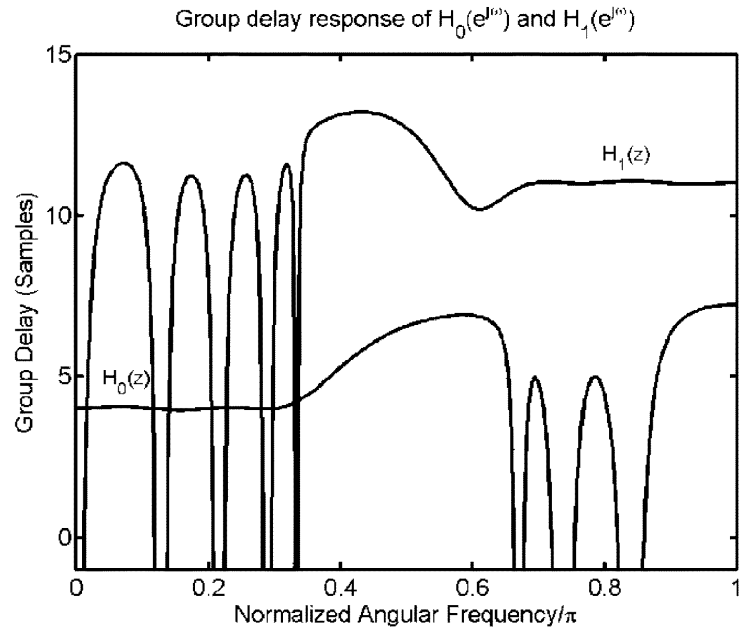

(c)
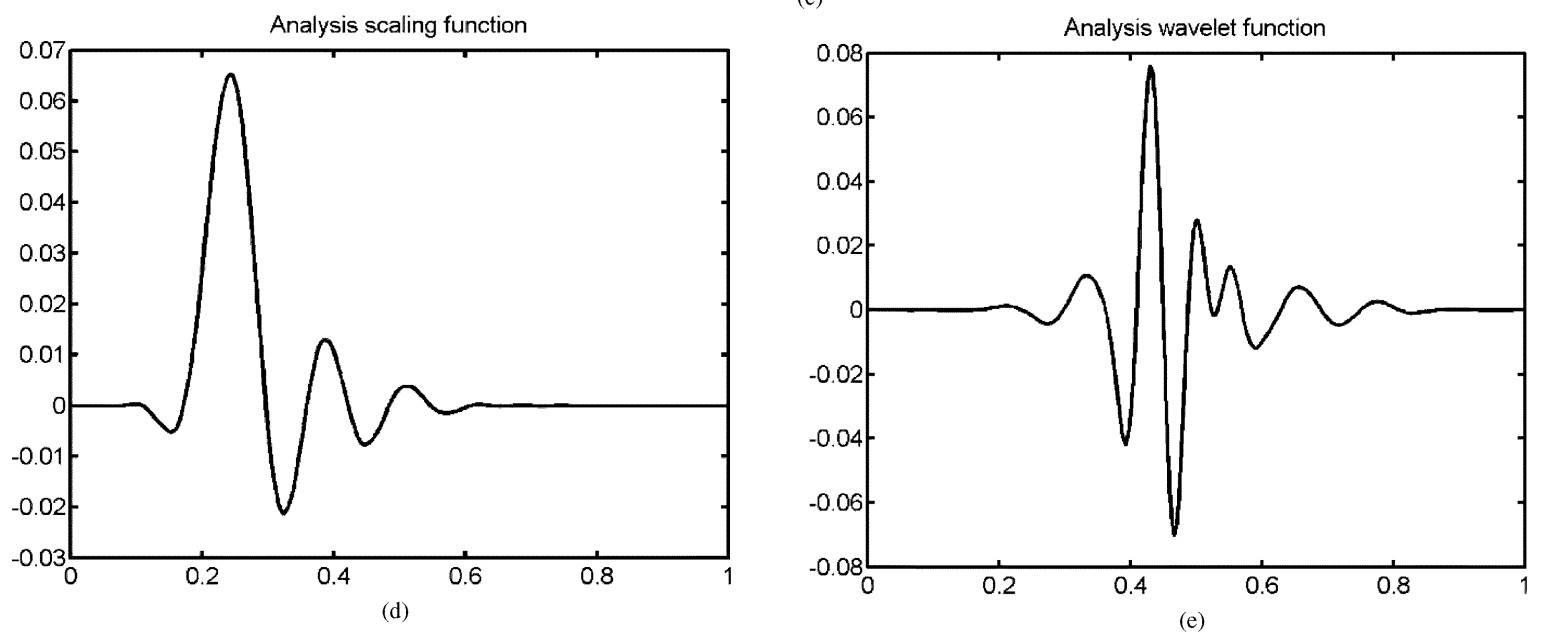

Fig. 2. Design results of Example 1. (a) Frequency response of the analysis filters pair designed by the QPLC method. (b) Frequency response and (c) group delay of the analysis filters pair designed by the SDP method. (d) Analysis scaling function. (e) Analysis wavelet function of the SDP design ( $\left.K_{0}=2, K_{1}=1\right)$.

Furthermore, it can be shown [25] that if $H_{0}(z)$ is a halfband filter, then the coefficients $b_{i}$ s also need to satisfy

$$
\begin{aligned}
b_{i} & =0, \text { for } i=0, \ldots, K-1 \\
b_{L-i} & =c_{L-i}, \text { for } i=0, \ldots, K-1 \\
\text { and } b_{i}-b_{L-i} & =c_{i}=-c_{L-i}, \text { for } \\
i & =K, K+1, \ldots,\left\lfloor\frac{L}{2}\right\rfloor .
\end{aligned}
$$

Since the coefficients of $\beta(z)$ are linear combination of the Bernstein coefficients $b_{i}$, therefore, if $b_{i} \mathrm{~s}$ are quantized to the SOPOT coefficient, so are the coefficients of $\beta(z)$. This also applies to $H_{\alpha}(z)$ and, hence, $\alpha(z)$. Therefore, it is possible to construct wavelet bases with SOPOT coefficients. Our approach is different from the one employed in [11]. Moreover, $H_{0}(z)$ and $H_{1}(z)$ are not restricted to being linear phase. Unfortunately, it was found that the wordlength of the filter coefficients is, in general, very long. In what follows, we propose another realiza- 


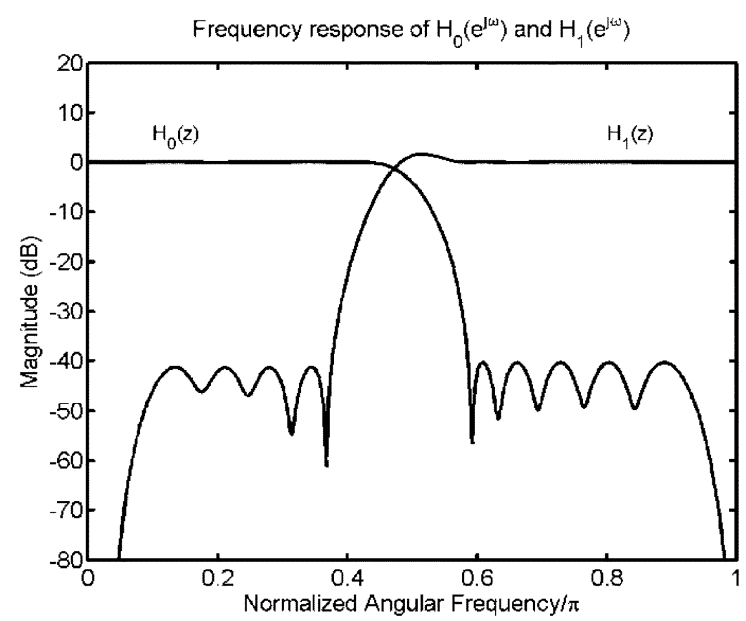

Fig. 3. Magnitude responses of analysis filters pair designed by the SDP method in example 2 .

tion, which provides a multiplier-less realization up to the fifth order of zero moments at a much lower coefficient wordlength. This factorization is an extension of the one that we have proposed in [2]. More precisely, the FIR function $\beta(z)$ is written as

$$
\beta(z)=R_{\beta}(z)+\left(1-z^{-1}\right)^{K} Q_{\beta}(z)
$$

where $R_{\beta}(z)=\sum_{m=0}^{K-1} r_{\beta, m} z^{-m}$ and $Q_{\beta}(z)=\sum_{n=0}^{L_{\beta}-K-1} q_{\beta, n} z^{-n}$ are, respectively, the remainder and quotient obtained by dividing $\beta(z)$ by $\left(1-z^{-1}\right)^{K}$. Let

$$
\begin{aligned}
& H_{0}(z)=\frac{\left[z^{-2 N}+z^{-1} \beta\left(z^{2}\right)\right]}{2}=z^{-1} H(z) \\
& H(z)=\frac{\left[z^{-2 N+1}+\beta\left(z^{2}\right)\right]}{2} .
\end{aligned}
$$

Using (14), we have

$$
H(z)=\left[z^{-2 N+1}+R_{\beta}\left(z^{2}\right)+\left(1-z^{-2}\right)^{K} Q_{\beta}\left(z^{2}\right)\right] / 2
$$

from which we see that if $H(z)$ contains the factor $\left(1 \pm z^{-1}\right)^{K}$, so does $z^{-2 N+1}+R_{\beta}\left(z^{2}\right)$. This is equivalent to the condition that the halfband filter $\left[z^{-2 N+1}+R_{\beta}\left(z^{2}\right)\right] / 2$ has maximal flatness at $z=-1$, and the coefficients of $R_{\beta}(z)$ are [12]:

$$
\begin{aligned}
r_{\beta, m} & =\frac{2^{-K+1}(-1)^{m} \prod_{i=0}^{K-1}(2 i-2 N+1)}{(2 m-2 N+1) m !(K-1-m) !} \\
m & =0,1, \ldots, K-1 .
\end{aligned}
$$

Once (15) is satisfied, the coefficients of $Q_{\beta}(z)$ can be quantized to arbitrary accuracy without violating the prescribed regularity condition. In [12], Pei et al. proposed another efficient form for implementing such maximally flat halfband filter. Instead of expanding the coefficients about $z^{-2}, R_{\beta}\left(z^{2}\right)$ is expanded as $R_{\beta}\left(z^{2}\right)=\sum_{n=0}^{K-1} b_{\beta, n}\left(z^{-2}-1\right)^{n}$, where the coefficients $b_{\beta, n}$ are given by $b_{\beta, 0}=0$ and $b_{\beta, n}=\left[\prod_{i=0}^{n-1}(2 N-1-i)\right] / 2^{n} n$ !, $n=1, \ldots, K-1$. It can be shown that $b_{\beta, n}$ and $r_{\beta, n}$ are related by $r_{\beta, m}=\sum_{n=0}^{K-1-m}{ }_{m} C_{n} \cdot b_{\beta, n}(-1)^{m-n}$, where ${ }_{m} C_{n}$ is the binomial coefficient. If the coefficients $b_{\beta, n}$ can be exactly represented as SOPOT coefficients, so can the coefficients $r_{\beta, m}$. In fact, this can be shown to be true for $n=0,1,2,3$, and 4: The case for $n=0,1$, and 2 follows directly because the denominators of $b_{\beta, n}$ are powers of two numbers.

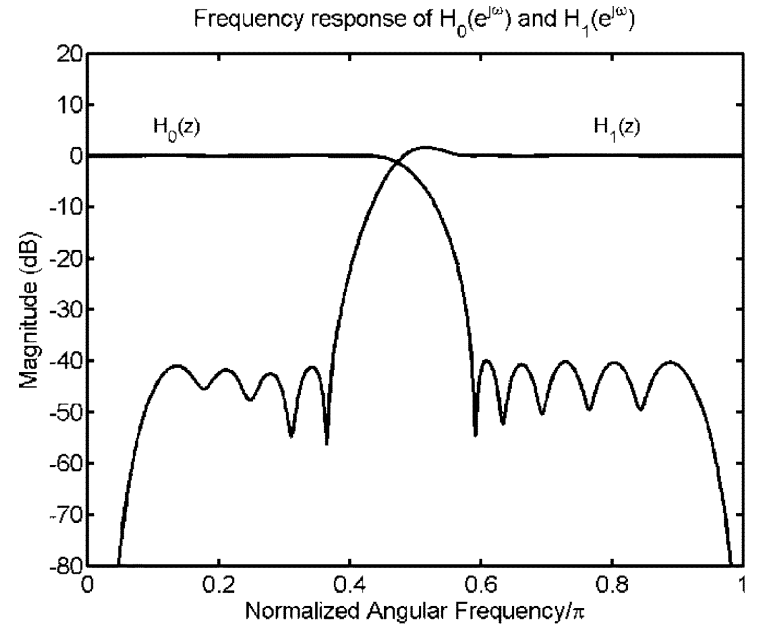

(a)

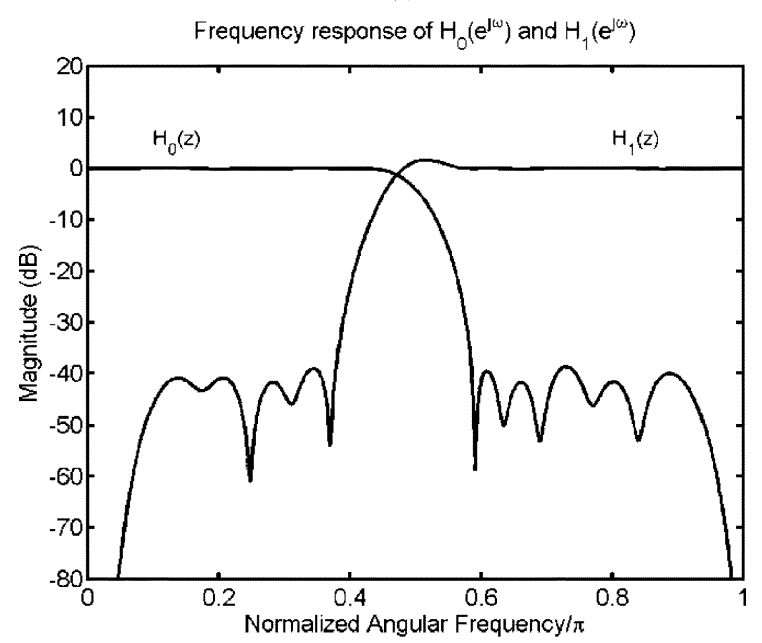

(b)

Fig. 4. Magnitude responses of SOPOT FB (analysis filters pair) derived from Example 2. (a) Using (13). (b) Using Bernstein polynomial expansion ( $K_{0}=$ $\left.3, K_{1}=3\right)$.

For $n=3$, the denominator of $b_{\beta, 3}=\left[\prod_{i=0}^{2}(2 N-1-i)\right] /\left(2^{4} 3\right)$ is a product of three consecutive odd numbers. Since at least one out of any three consecutive odd numbers must contain a factor of 3 , it follows that $\left[\prod_{i=0}^{2}(2 N-1-i)\right] / 3$ is an integer. As $b_{\beta, 4}=(2 N-1-3) b_{\beta, 3} / 2^{3}$, it is also a SOPOT coefficient. $\alpha(z)$ can also be realized using this method. Multiplier-less IIR wavelet bases can readily be obtained from their FIR counterparts by model reducing the quotient $Q(z)$ to $\hat{Q}(z)$, and quantizing the coefficients of $\hat{Q}(z)$ to SOPOT coefficients [25]. To obtain a multiplier-less realization of the structural PR FB, the filter coefficients of $Q_{\beta}(z)$ and $Q_{\alpha}(z)$ are represented as SOPOT or canonical signed digit (CSD) coefficients. To further reduce the number of adders in realizing $Q_{\beta}(z)$ and $Q_{\alpha}(z)$, they are implemented in transposed form using the technique of MB [13]. MB is a very useful technique to reduce the number of adders needed to implement the multiplications of an integer input with a set of constant integer coefficients. In principle, it is possible to remove all the redundancy found in the constant multipliers leading to a realization with a minimum number of adders. Therefore, the implementation complexity of the structural PR FB can be drastically reduced (around $40 \%$ in our example). The design of multiplier-less digital filters and FBs is an important and active topic of research [2]-[4], [6], [17], [18], [20]-[23]. In this paper, the random search algorithm proposed in [4] is employed because of its simplicity and effectiveness. Due to page limitations, details are omitted. For the 
TABLE I

SOPOT COEFFICIENT OF $Q_{\beta}(z)$ AND $Q_{\alpha}(z)$ IN EXAMPLE 3. TOTAL NUMBER OF ADDERS FOR $Q_{\beta}(z)$ AND $Q_{\alpha}(z)$ ARE, RESPECTIVELY, 26 AND 21 WITHOUT USING MB

\begin{tabular}{|c|c|c|}
\hline$n$ & $Q_{\beta}(n)$ & $Q_{\alpha}(n)$ \\
\hline 0 & $-2^{1}+2^{-3}-2^{-6}-2^{-8}-2^{-10}-2^{-12}$ & $-2^{2}-2^{-1}+2^{-3}+2^{-7}-2^{-10}$ \\
\hline 1 & $-2^{-1}+2^{-3}-2^{-11}-2^{-13}$ & $-2^{1}+2^{-3}+2^{-9}-2^{-11}$ \\
\hline 2 & $2^{-4}-2^{-6}+2^{-10}-2^{-12}$ & $-2^{-2}-2^{-3}+2^{-5}+2^{-9}-2^{-11}$ \\
\hline 3 & $-2^{-4}+2^{-8}+2^{-11}$ & $2^{-4}+2^{-6}+2^{-8}+2^{-10}$ \\
\hline 4 & $2^{-7}-2^{-9}+2^{-11}-2^{-13}$ & $-2^{-6}-2^{-7}+2^{-9}$ \\
\hline 5 & $-2^{-5}+2^{-8}-2^{-11}$ & $2^{-5}-2^{-8}-2^{-10}$ \\
\hline 6 & $2^{-7}+2^{-9}-2^{-12}$ & $-2^{-7}-2^{-10}$ \\
\hline 7 & $-2^{-7}+2^{-10}+2^{-12}$ & $2^{-7}-2^{-9}$ \\
\hline 8 & $2^{-6}-2^{-9}-2^{-11}$ & $-2^{-8}-2^{-10}$ \\
\hline 9 & $2^{-8}-2^{-10}-2^{-13}$ & \\
\hline 10 & $2^{-7}$ & \\
\hline
\end{tabular}

problem considered here, the overall design time is less than $10 \mathrm{~min}$ using a Pentium-533 PC with Matlab 5.3.

Example 3: In this example, a two-channel multiplier-less PR FIR FB with $K_{0}=K_{1}=3$ is designed. The specification is identical to Example 2. With the use of (13), the remainder polynomials are found to be $R_{\beta}(z)=(15 / 8)-(21 / 4) z^{-1}+(35 / 8) z^{-2}$ and $R_{\alpha}(z)=$ $(35 / 8)-(45 / 4) z^{-1}+(63 / 8) z^{-2}$. Since the denominators of the polynomial coefficients are powers of 2 , they can be exactly implemented as SOPOT coefficient. The optimized SOPOT quotients $Q_{\beta}(z)$ and $Q_{\alpha}(z)$ are given in Table I, and the magnitude response of the multiplier-less FB is shown in Fig. 4(a). From the pole-zero plot (not shown here), it is observed that the regularity condition is preserved. The number of adders required to synthesize $Q_{\beta}(z)$ and $Q_{\alpha}(z)$, without using $\mathrm{MB}$, are 26 and 21, respectively. Using MB, the number of adders is reduced to 14 and 11 , respectively. It can also be seen that the optimized frequency response is approximately equiripple in the stopband with an approximately linear phase at the passband. The stopband attenuation is around $40 \mathrm{~dB}$, which is comparable with the result of design Example 2. The corresponding analysis scaling and wavelet functions are also very smooth (not shown here). For comparison purposes, we also designed a multiplier-less FB using the Bernstein polynomial expansion described earlier. We first expanded the halfband filters $H(z)$ and $H_{\alpha}(z)$ and retained the integer bits [the $b_{i} \mathrm{~s}$ in (12)] of their corresponding Bernstein coefficients. Then, the SOPOT coefficients of $\beta(z)$ and $\alpha(z)$ are extracted. The wordlength of the coefficients (not shown here due to page limitations) has a smallest power of two term of $2^{-20}$, which is significantly longer than that in Table I (the smallest power of two term is $2^{-13}$ ). The frequency response of the FB using the Bernstein polynomial expansion approach is shown in Fig. 4(b). Compared with the frequency response in Fig. 3, more degradation is observed even at a much longer wordlength.

\section{CONCLUSIONS}

Methods for designing a class of two-channel PR FIR FBs and wavelets in [1] and [2] are presented. The $K$-regularity conditions are expressed as a set of linear equality constraints, and the design problem is solved as either a QPLC or a SDP problem. It is also shown by means of Bernstein polynomial expansion that wavelet FBs with SOPOT coefficients always exist. Unfortunately, this structure requires relatively long coefficient wordlength, and another hardware efficient structure is proposed, which is able to realize wavelets FB up to the fifth-order zero moment. Design and efficient realizations of these multiplier-less FBs are also presented.

\section{APPENDIX}

The analysis and synthesis filters $H_{i}(z)$ and $F_{i}(z)$ of the structural two-channel FB satisfy the following:

Analysis

$$
\begin{aligned}
& H_{0}(z)=\frac{\left[z^{-2 N}+z^{-1} \beta\left(z^{2}\right)\right]}{2} \\
& H_{1}(z)=-\alpha\left(z^{2}\right) H_{0}(z)+z^{-2 M-1}
\end{aligned}
$$

Synthesis

$$
F_{0}(z)=-H_{1}(-z) \text { and } F_{1}(z)=H_{0}(-z) \text {. }
$$

Phoong [1, eqn.10] showed that for $\alpha(z)=\beta(z)$ and $M=2 N-1$, $F_{0}(z)$ and $H_{1}(z)$ satisfy

$$
\begin{aligned}
& F_{0}(z)=\left(2 z^{-2 N+1}-\beta\left(z^{2}\right)\right) H_{0}(z) \\
& H_{1}(z)=\left(2 z^{-2 N+1}+\beta\left(z^{2}\right)\right) F_{1}(z) .
\end{aligned}
$$

It can be seen from (A.3) that the zeros of $H_{0}(z)$ are also the zeros of $F_{0}(z)$. For wavelet FBs, both $H_{0}(z)$ and $F_{0}(z)$ should have certain zeros at $z=-1$. It then follows for the structural PR FB with $\alpha(z)=$ $\beta(z)$ that if $H_{0}(z)$ has $K_{0}$ zeros at $z=-1$, then so does $F_{0}(z)$. Thus, wavelet FBs can simply be generated by imposing $K_{0}$ zeros at $z=$ -1 for $H_{0}(z)$. Another observation is that because of the relationship between $F_{0}(z)$ and $H_{1}(z), H_{1}(z)$ will have $K_{0}$ zeros at $z=1$. We now consider the general case where $\alpha(z)$ is not equal to $\beta(z)$. From (A.1), we have, after some manipulation, the following:

$$
\begin{aligned}
F_{0}(z) & =-H_{1}(-z)=\alpha\left(z^{2}\right) H_{0}(-z)+z^{-2 M-1} \\
& =-\alpha\left(z^{2}\right) H_{0}(z)+2 H_{\alpha}(z) \cdot z^{-2 N+1}
\end{aligned}
$$

where $2 M+1=2 N_{\Delta}+2 N-1$ or $N_{\Delta}=(M-N)+1$, and $H_{\alpha}(z)=\left[z^{-1} \alpha\left(z^{2}\right)+z^{-2 N_{\Delta}}\right] / 2$ is a halfband filter. If $H_{0}(z)$ and $H_{\alpha}(z)$ have $K_{1}$ common zeros at $z=-1$, then $F_{0}(z)$ will have at least $K_{1}$ zeros at $z=-1$. Therefore, a sufficient condition for the latter is to impose $K_{1}$ zero on $H_{\alpha}(z)$ at $z=-1$ and choose $K_{0} \geq K_{1}$. This property also applies to the case of general FIR or irreducible IIR functions $\alpha(z)$ and $\beta(z)$.

\section{REFERENCES}

[1] S. M. Phoong, C. W. Kim, P. P. Vaidyanathan, and R. Ansari, "A new class of two-channel biothogonal filter banks and wavelet bases," IEEE Trans. Signal Processing, vol. 43, pp. 649-664, Mar. 1995.

[2] J. S. Mao, S. C. Chan, W. Liu, and K. L. Ho, "Design and multiplier-less implementation of a class of two-channel PR FIR filterbanks and wavelets with low system delay," IEEE Trans. Signal Processing, vol. 48, pp. 3379-3394, Dec. 2000.

[3] W. Liu, S. C. Chan, and K. L. Ho, "Low-delay perfect reconstruction two-channel FIR/IIR filter banks and wavelet bases with SOPOT coefficients," in Proc. IEEE ICASSP 2000, Istanbul, Turkey, May 2000.

[4] C. K. S. Pun, S. C. Chan, and K. L. Ho, "Efficient design of a class of multiplierless perfect reconstruction two-channel filter banks and wavelets with prescribed output accuracy," in Proc. 11th IEEE Signal Process. Workshop Statistical Signal Process., 2001, pp. 599-602.

[5] S. C. Chan, C. K. S. Pun, and K. L. Ho, "The design of a class of perfect reconstruction two-channel FIR and wavelets filterbanks using constrained least squares method and semidefinite programming," in Proc. 14th Int. Conf. Digital Signal Process., Santorini, Greece, July 2002.

[6] S. Sriranganathan, D. R. Bull, and D. W. Redmill, "The design of low complexity two-channel lattice-structure perfect-reconstruction filter banks using genetic algorithms," in Proc. IEEE ISCAS, vol. 4, June 1997, pp. 2393-2396.

[7] K. Steiglitz, T. W. Parks, and J. F. Kaiser, "METEOR: A Constraintbased FIR filter design program," IEEE Trans. Signal Processing, vol. 40, pp. 1901-1909, Aug. 1992. 
[8] M. J. T. Smith and T. P. Barnwell III, "Exact reconstruction for treestructured subband coders," IEEE Trans. Acoust., Speech, Signal Processing, vol. ASSP-34, pp. 431-441, June 1986.

[9] P. Duhamel and O. Rioul, "A remez exchange algorithm for orthonormal wavelets," IEEE Trans. Circuits Syst. II, vol. 41, pp. 550-560, Aug. 1994.

[10] I. Daubechies and W. Sweldens, "Factoring wavelet transform into lifting steps," J. Fourier Anal. Appl., vol. 4, no. 3, pp. 247-269, 1998.

[11] D. B. H. Tay, "Two stage, least squares design of biorthogonal filter banks," in Proc. IEEE ISCAS, vol. 1, 2000, pp. 591-594.

[12] S. C. Pei and P. H. Wang, "Closed-form design and efficient implementation of generalized maximally flat half-band FIR filters," IEEE Signal Processing Lett., vol. 7, pp. 149-151, June 2000.

[13] A. G. Dempster and M. D. Macleod, "Use of minimum-adder multiplier blocks in FIR digital filters," IEEE Trans. Circuits Syst. II, vol. 42, pp. 569-577, Sept. 1995.

[14] W. S. Lu and A. Antoniou, "Design of digital filters and filter banks by optimization: A state of the art review," in Proc. EUSIPCO, Tampere, Finland, Sept. 2000

[15] L. Vandenberghe and S. Boyd, "Semidefinite programming," SIAM J. Rev., vol. 38, pp. 49-95, Mar. 1996.

[16] R. Fletcher, Practical Methods of Optimization, 2nd ed. Chichester: John Wiley \& Sons, 1987.

[17] Y. C. Lim and S. R. Parker, "FIR filter design over a discrete power-of-two coefficient space," IEEE Trans. Acoust. Speech, Signal Processing, vol. ASSP-31, pp. 583-519, June 1983.
[18] J. S. Mao, W. S. Lu, S. C. Chan, and A. Antoniou, "Design and multiplierless implementation of two-channel biorthogonal IIR filter banks with low system delay," Proc. ISCAS, vol. 2, pp. 465-468, 2001.

[19] S. Samadi, A. Nishihara, and H. Iwakura, "Universal maximally flat lowpass FIR systems," IEEE Trans. Signal Processing, vol. 48, pp. 1956-1964, July 2000.

[20] Y. M. Hasan, L. J. Karam, M. Falkinburg, A. Helwig, and M. Ronning, "Canonic signed digit chebyshev FIR filter design," IEEE Signal Processing Lett., vol. 48, pp. 167-169, June 2001.

[21] H. Samueli, "An improved search algorithm for the design of multiplierless FIR filters with power-of-two coefficients," IEEE Trans. Circuits Syst., vol. 36, pp. 1044-1047, Dec. 1989.

[22] C. L. Chen and A. N. Willson Jr, "A trellis search algorithm for the design of FIR filters with signed-power-of-two coefficients," IEEE Trans. Circuits Syst. II, vol. 46, pp. 29-39, Jan. 1999.

[23] N. Benvenuto, M. Marchesi, and A. Uncini, "Application of simulated annealing for the design of special digital filters," IEEE Trans. Signal Processing, vol. 40, pp. 323-332, Jan. 1992.

[24] S. C. Chan, Carson, K. S. Pun, and K. L. Ho, "The design of a class of perfect reconstruction two-channel FIR linear-phase filterbanks and wavelets bases using semideginite programming," IEEE Signal Processing Lett., to be published.

[25] Carson and K. S. Pun, "New design and realization techniques for perfect reconstruction two-channel filterbanks and wavelets bases," M.Phil. thesis, Univ. Hong Kong, 2002. 